\title{
Antimicrobial stewardship: Fundamental human responsibility
}

\section{Thapa B}

Badri Thapa, Public Health Specialist, Bishal Nagar, Kathmandu, Nepal

I n 2015, world leaders committed to achieve 17 ambitious and far-reaching Sustainable Development Goals (SDGs) by 2030.The Goals are interconnecting to ensure 'leave no one behind' by 2030. Of these goals, SDG 3 is to "ensure healthy lives and promote well-being for all at all ages". Achieving Universal Health Coverage (UHC) is one among 8 pillars of SDG 3. "Health for all" has therefore been the guiding vision of World Health Organization (WHO) for more than seven decades to support countries in moving towards UHC. One of the important aspects is to have access to essential medicines. Ensuring future generations have access to antimicrobials is also high on agenda for many heads of state, and almost all Ministers of Health. On 21 September 2016, during the $71^{\text {st }}$ Session of the United Nations General Assembly, Member States adopted the Political Declaration on High-level Meeting on Antimicrobial Resistance (AMR). An ad hoc Interagency Coordination Group on AMR was set up, ${ }^{1}$ in consultation with the tripartite agencies- WHO, Food and Agriculture Organization (FAO) of the United Nations, and the World Organization for Animal Health with the objective to provide practical guidance for approaches needed to ensure sustained effective global action to address AMR, including options to improve coordination, considering the Global Action Plan on AMR.

The magnitude of AMR problem is now well accepted by the countries. With no or limited potential solutions to slow down, rising trends of drug resistance will cost an estimated 10 million lives a year and a cumulative 100 trillion USD of economic output are at risk by $2050^{2}$. It is in policy makers and governments' hands to take steps to change this situation. Because microbes have no borders, some of the steps that are required will need to

Address for correspondence

Dr. Badri Thapa

Public Health Specialist

Bishal Nagar, Kathmandu, Nepal

E-mail: badri_bishal@yahoo.com be addressed internationally. What is certain is that no single sector in a country can solve the AMR problem on its own. Solutions to address AMR must have universal access to healthcare and essential antimicrobials at their heart and they must stop wasting antimicrobials that are effective.

Many infectious disease pathogens are demonstrating increasing levels of resistance to commonly used antibiotics $^{3}$. A universal rise of drug-resistant infections could be managed through addressing the antibiotics supply and demand sides. The supply of new antimicrobials is insufficient to keep pace with the increase in drug resistance. Older generations of antimicrobials are used more widely and microbes evolve to resist them. At the same time, the demand for these medicines is very badly managed: huge quantities of antimicrobials-in particular antibiotics-are wasted globally on patients, animals and agriculture who do not need them while others who need them do not have access. Countries have important role to play to reduce the demand for antibiotics in healthcare systems, pharmaceutical industry and agriculture and food production industry ${ }^{2}$.The demand of these antibiotics could be reduced through wider health literary promotion on antibiotic use so that patients and farmers do not demand and clinicians and veterinarians do not prescribe more than in need.

Patient safety practices in hospitals through hand washing, access to clean water and sanitation-is the key to prevent the spread of drug resistant nosocomial infections. Less the people are infected, less is the need for the antibiotics. On the other hand, antibiotics are used simply as a growth promoter in agriculture, aquaculture, and livestock promoting AMR. Drugresistant pathogens and their resistant genes are now considered as environment pollutants infecting water sources through hospital waste that can reach from farm-to-fork. The antibiotics that are considered last resort for humans are widely used in animal husbandry. 
United States Food and Drug Administration estimates over 70 percent (by weight) are sold for use in animals. Hence, there is a dire need to reduce the demand and use of antibiotics in these sectors.

There is a need for a robust national surveillance to monitor antimicrobial consumption in humans, animals and agriculture. The data on drug resistance generates evidence that can formulate policies for AMR. This has been evidenced by development of the Global Health Security Agenda (GHSA)-AMR is part of it-, the announcement of 375 million USD Fleming Fund for AMR by United Kingdom (UK) government ${ }^{4}$ and the development of Global AMR Surveillance System (GLASS) by WHO. Research is another component, where new and rapid diagnostics could create less demand for antibiotics. This could transform the way we use antimicrobials in humans and animals. Finally, vaccination has been proven most effective public health intervention that can decrease the infection creating less demand for the antibiotics.

Antibiotics are a special category of antimicrobial drugs without which key medical procedures (gut surgery, caesarean sections, joint replacements, and treatments that depress the immune system, such as chemotherapy for cancer) could become too dangerous to perform. At the same time, most of the drug resistant infections are not treatable. We now know that 700,000 people die of resistant infections every year. Most of direct and much of indirect impact of AMR will fall on low income country like Nepal.

The 'WHO Model essential medicines list (EML)'was launched in 1977, coinciding with the endorsement by governments at the World Health Assembly of "Health for all" as the guiding principle for WHO and countries' health policies. Many countries have adopted the concept and have developed lists of their own, using the WHO EML as a guide. The EML is updated and revised every two years by the WHO Expert Committee. The $20^{\text {th }}$ Essential Medicines List published on 6 June 2017 marks the $40^{\text {th }}$ anniversary of this flagship WHO tool to expand access to medicines ${ }^{5}$. The updated list adds 30 medicines for adults and 25 for children, and specifies new uses for 9 already-listed products, bringing the total to 433 drugs deemed essential for addressing the most important public health needs globally. The list also provides new advice on which antibiotics to use for 21 most common infections and which to preserve for the most serious syndromes, based on a thorough review of all essential antibiotics. The List categorizes antibiotics into three groups- ACCESS, WATCH, and RESERVE- to optimize antibiotic use and reduce antibiotic resistance without restricting access:

- The ACCESS group to be available at all times as treatments for a wide range of common infections. The drugs that fall under this category include drugs such as Amoxicillin which is widely-used for treating infections such as pneumonia;

- The WATCH, includes antibiotics that are recommended as first or second choice treatments for a small number of infections. For example- the use of Ciprofloxacin is used to treat cystitis (a type of urinary tract infection) and upper respiratory tract infections should be dramatically reduced to avoid further development of resistance; and

- The RESERVE includes antibiotics such as Colistin and some Cephalosporin that should be considered last options, and used only in the most severe circumstances when all other alternatives have failed such as for life-threatening infections due to multidrug resistant bacteria.

With the federalism in the health sector in Nepal, each level of governance (Federal, Provincial and Local) has the authority to procure antimicrobials. This poses greater risk on ensuring the quality of antimicrobials and their rational use. Therefore, it is essential for the Ministry of Health and Population to revisit its EML in line with WHO recommendation and clearly identify which level of government should buy which drugs. The recently endorsed Public Health Act 2018 bans sale of drugs without prescription and promotes generic prescription. Ensuring the implementation of these regulations at each level of government with stringent monitoring is crucial to combat AMR. It is more important to implement WHO EML recommendation on antibiotics in Nepal.

Improving the pay, recognition, identifying the career paths and rewards to medical professionals- doctors and nurses working in the infectious disease units and pharmacists could widely practice antibiotic stewardship as categorized in $20^{\text {th }}$ WHO EML. Finally, 'Health is a fundamental human right'. At the same time, 'Preventing the emergence and spread of $A M R$ is a fundamental responsibility of every human being'. Let's work together to provide access to the effective antibiotics to our next generations. 


\section{REFERENCES}

1. Rochford C, Sridhar D, Woods N, Saleh Z, Hartenstein L, Ahlawat $\mathrm{H}$ et al. Global governance of antimicrobial resistance. The Lancet [Internet]. 2018[Cited 2018 June 15]; 391(10134):1976-78. [DOI]

2. O'Neill J. Tackling drug-resistant infections globally: Final report and recommendations, the review on antimicrobial resistance [Internet]. 2016. [Cited 2018 June 15].[Full text]
3. Pourmand A, Mazer-Amirshahi M, Jasani G, May L. Emerging trends in antibiotic resistance: Implications for emergency medicine. Am J Emerg Med [Internet]. 2017[Cited 2018 June 18];35(8):1172-76. [DOI]

4. UK Government. The Fleming Fund [Internet]. 2018[Cited 2018 May 18]. [Website]

5. World Health Organization. WHO Model List of Essential Medicines.20 ${ }^{\text {th }}$ List [Internet]. 2017. [Cited 2018 June 20]. [Full text] 